\title{
A single natural image denoising based on independent component analysis
}

\author{
Mr.VipulPatil ${ }^{1}$, Dr. A.J. Patil ${ }^{2}$ \\ Student, Department of Electronics and Communication, Shri.Gulabrao Deokar C.O.E., Jalgaon, India ${ }^{1}$ \\ Professor, Department of Electronics and Communication, Shri.Gulabrao Deokar C.O.E., Jalgaon, India ${ }^{2}$
}

\begin{abstract}
Denoising of natural image is challenging problem in image processing. Image denoising problem is very simple. Independent component analysis (ICA) is higher order statistical tool for analysis of multidimensional data. Image denoising using independent component analysis assume an Gaussian noise. The noise is a Gaussian and considered image data is non-Gaussian random variable. In practical situations type of noise, type of image, amount of noise are all variable parameters. Such a parameter cannot effectively remove the noise. In this paper we use a independent component analysis to remove a noise from a single image. Natural images provide the basic knowledge for understanding.
\end{abstract}

Keywords: Adaptive PCA, Denoise, ICA, PCA.

\section{INTRODUCTION}

Image denoising is an essential part in an image processing field. Denoising of image is a research problem and a challenging work in image processing because image have variable parameters such as type image, type of noise and amount of noise in images. Denoising techniques such as Fourier transform and wavelet transform method both are not data adaptive methods these are localized in frequency domain and spatial domain.( ie.,[1])A data adaptive comes out from filtering approach that results in a inherent property of ICA techniques. Data adaptive is a very important part in the denoising of image.(ie.,[2]). For this method considered image are corrupted by Gaussian noise. We compare peak single to noise ratio (PSNR) of denoised and noisy image of various algorithms. The following denoised methods are discussed to understand denoising of natural image.

1.Principle component analysis.

2. Adaptive component analysis.

3. Independent component analysis.

\section{PRINCIPAL COMPONENT ANALYSIS}

Principal Component Analysis (PCA) is an exploratory tool designed by Karl Pearson in 1901 to identify unknown trends in a multidimensional data set $\mathbf{X}$. The algorithm was introduced to psychologists in 1933 by $\mathrm{H}$. Hotelling, hence sometimes it is called Hotelling's Transform. However, today we know that implementing PCA is the equivalent of applying Singular Value Decomposition (SVD) on the covariance matrix of a data set $(1.2,1.3)$. By providing a tutorial on PCA using SVD, students are familiarized with both matrix decomposition techniques. When there is need to dimension reduction (reduce the no. of variables) and further analyse the relationship between different variables (quantity); we can use PCA to solve this kind of problems. Principal components are the direction of greatest

Variability (covariance) in the data, then the next orthogonal (uncorrelated) direction of the greatest variability. So first remove all the variability along the first component, and then find the next direction of greatest variability and so on....

The mathematical calculation for this is as given below(ie.,[3]) PCA is the evident from the two projections, that what we see depends on the direction onto which we project.

The mean can be calculate by using the formula-[3]

$\bar{x}=\frac{1}{n} \sum_{i=1}^{n} x_{i}$

The variance can be calculate by using-

$\sigma^{2}=\frac{1}{n} \sum_{i=1}^{n}\left(x_{i}-\bar{x}\right)^{2}$

To find the direction $\hat{u}$, such that projecting $\mathrm{n}$ points in the d dimensions, $\vec{e}^{i}, \mathrm{i}=1,2 \ldots \ldots, \mathrm{n}$, onto $\hat{u}$, gives the largest variance.(ie.,[3])

To construct the covariance matrix-

$c_{i j}=\frac{1}{n} \sum_{k=1}^{n}\left(e_{i}^{k}-\vec{e}_{i}\right)\left(e_{j}^{k}-\vec{e}_{j}\right)$

$\hat{u}$ is the eigenvector of $\mathrm{c}$ with the largest eigenvalue:

$$
\mathrm{C} \hat{u}=\lambda_{1} \hat{u}
$$

To show that the variance of the projections is indeed $\lambda_{1}$, we first calculate our sample vectors their projections onto û: [3]

$e_{u}^{k}=\vec{e}^{(k)} \cdot \hat{u}=\sum_{i=1}^{d} e_{i}^{(k)} \hat{u}_{i}$

The total variance of the data is, [3]

$\sigma^{2}=\sum_{k}\left(\vec{e}^{(k)}-\bar{e}\right)^{2}$

Is also given by [3] the sum of the variance of the projections on the principal components $\sigma^{2}=\sum_{\alpha=1}^{d} \sigma_{\alpha}^{2}=\sum_{\alpha} \lambda_{\alpha}$

The fraction of the total variance that has been captured is:[3]

$\sigma^{\text {captured }}=\sum_{\propto=1}^{d} \frac{\sigma_{\alpha}^{2}}{\sigma^{2}}($ ie.,,$[3])$ 


\section{INDEPENDENT COMPONENT ANALYSIS}

ICA is nothing but the independent component analysis, as it's name implies it is the method for finding underlying factors or components from multi dimensional statistical data. What distinguishes ICA from other methods is that it looks for components that are both statistically independent and non-Gaussian. Blind source separation or ICA is the identification and separation of mixtures of sources with little prior information. Multiple sources human being can focus on the interested source/sources, but hard to separate by signal processing techniques, then we can use independent component analysis to solve this problem. A speech source signal $s 1$ is represented as $s 1=$ $(s 11, s 21, \ldots, s N 1)$, where $s 1$ adopts amplitudes $s 11$, then 21 , and so on; superscripts specify time and subscripts specify signal identity (e.g., speaker identity).We will be considering how to mix and unmix a set of two or more signals, and we define a specific set of two time varying speech signals $s 1$ and $s 2$ in order to providea concrete example. Now, the amplitudes of both signals can be written as a vector variable $\mathbf{s}$, which can be rewritten in one of several mathematically equivalent forms:(ie.,[5]).

$$
\begin{aligned}
& \mathrm{S}=\left(\begin{array}{l}
s_{1} \\
s_{2}
\end{array}\right) \\
& =\left(\begin{array}{c}
\left(s_{1}^{1}, s_{1}^{2}, \ldots \ldots \ldots . . s_{1}^{N}\right) \\
\left(s_{2}^{1}, s_{2}^{2}, \ldots \ldots \ldots \ldots . . s_{2}^{N}\right)
\end{array}\right)
\end{aligned}
$$

The Mixing and UnmixingMatrices(ie.,[5])

The set of mixtures defines a vector variable $\mathbf{x}=(x 1, x 2) T$, and the transformation from $\mathbf{s}$ to $\mathbf{x}$ definesa mixing matrix A:

$$
\mathrm{X}=\left(\begin{array}{ll}
a & b \\
c & d
\end{array}\right)\left(\begin{array}{c}
s_{1}^{1}, s_{1}^{2}, \ldots \ldots \ldots \ldots s_{1}^{N} \\
s_{2}^{1}, s_{2}^{2}, \ldots \ldots \ldots \ldots . . s_{2}^{N}
\end{array}\right)
$$

The mapping from $\mathbf{x}$ to $\mathbf{s}=(s 1, s 2) T$ defines an optimal unmixing matrix $\mathbf{W} *=(\mathbf{w} 1, \mathbf{w} 2) T$ with (row) weight vectors $\mathbf{w} T 1=(\alpha, \beta)$ and $\mathbf{w} T 2=(\gamma, \delta)$

$$
\begin{aligned}
& \mathbf{S}=\left(\begin{array}{ll}
\alpha & \beta \\
\gamma & \delta
\end{array}\right)\left(\begin{array}{l}
x_{1}^{1}, x_{1}^{2}, \ldots \ldots \ldots \ldots \ldots \ldots . . . x_{1}^{N} \\
x_{2}^{1}, x_{2}^{2}, \ldots \ldots \ldots \ldots \ldots \ldots x_{2}^{N}
\end{array}\right) \\
& =\left(w_{1}, w_{2}\right)^{T}\left(x_{1}, x_{2}\right) \\
& =w^{*} \mathrm{X}
\end{aligned}
$$

It can be seen that $\mathbf{W} *$ reverses, or inverts, the effects of $\mathbf{A}$, and indeed, $\mathbf{W} *$ could be estimated from the matrix inverse $\mathbf{W} *=\mathbf{A}-1$, if $\mathbf{A}$ were known3. However, as we are ultimately concerned with finding $\mathbf{W} *$ when $\mathbf{A}$ is not known, we cannot, therefore, use $\mathbf{A}-1$ to estimate $\mathbf{W} *$. For arbitrary values of the unmixing coefficients, the unmixing matrix is suboptimal and is denoted $\mathbf{W}$. In this case, the signals extracted by Ware not necessarily source signals, and are denoted $\mathbf{y}=\mathbf{W x}(\mathrm{ie} .,[5])$. Consider a (mixture) vector variable $\mathbf{x}$ with joint $\operatorname{pdf} p \mathbf{x}$, and a (source) vector variable $\mathbf{s}$ with joint $\operatorname{pdf} p \mathbf{s}$, such that $\mathbf{s}=\mathbf{W} * \mathbf{x}$, where $\mathbf{W} *$ is the optimal unmixing matrix. As noted above, the number of source signals and mixtures must be equal, which ensures that $\mathbf{W} *$ is square. In general, the relation between the joint pdfs of $\mathbf{x}$ and $\mathbf{s}$ is[5]

$$
p_{x}(X)=p_{s}(s)\left|w^{*}\right|
$$

where $|\mathbf{W} *|=|\partial \mathbf{s} / \partial \mathbf{x}|$ is the Jacobianof $\mathbf{s}$ with respect to $\mathbf{x}$. Equation (3.7) defines the likelihood of the observed mixtures $\mathbf{x}$, which is the probability of $\mathbf{x}$ given $\mathbf{W} *$. For any non optimalunmixing matrix $\mathbf{W}$, the extracted signals are $\mathbf{y}=\mathbf{W} \mathbf{x}$. Making the dependence on $\mathbf{W}$ explicit, the likelihood $p \mathbf{x}(\mathbf{x} \mid \mathbf{W})$ of the signal mixtures $\mathbf{x}$ given $\mathbf{W}$ is [5] $p_{x}(x \mid W)=p_{s}\left(W_{x}\right)|W|$

We would naturally expect $p \mathbf{x}(\mathbf{x} \mid \mathbf{W})$ to be maximal if $\mathbf{W}=$ $\mathbf{W} *$. Thus, (3.8) can be used to evaluate the quality of any putative unmixing matrix $\mathbf{W}$ in order to find that particular $\mathbf{W}$ that maximizes $p \mathbf{x}(\mathbf{x} \mid \mathbf{W})$. By convention, (3.8) defines a likelihood function $L(\mathbf{W})$ of $\mathbf{W}$, and its logarithm defines the log likelihood function $\ln L(\mathbf{W})$. If the $M$ source signals are mutually independent, so that the joint pdf $p$ sisthe product of its $M$ marginal pdfs, then can bewritten (ie.,[5]). $\ln L(\mathrm{~W})=\sum_{i}^{M} \sum_{t}^{N} \ln p_{s}\left(W_{i}^{T} x^{t}\right)+\mathrm{N} \ln |W|$

Note that the likelihood $L(\mathbf{W})$ is the joint $\operatorname{pdf} p \mathbf{x}(\mathbf{x} \mid \mathbf{W})$ for $\mathbf{x}$, but using MLE, it is treated as if it were a function of the parameter $\mathbf{W}$. If we substitute a commonly used leptokurtotic model joint pdf for the source signals $p \mathbf{s}(\mathbf{y})=$ $(1-\tanh (\mathbf{y}) 2)$, then $\operatorname{Ln} \quad \mathrm{L}(\mathrm{W})=\sum_{i}^{M} \sum_{t}^{N} \ln (1-$ tanhigi $(w i T x t) 2+N \ln (3.10)$

The matrix $\mathbf{W}$ that maximizes this function is the maximum likelihood estimateof the optimal unmixing matrix $\mathbf{W} *$. Equation (3.9) provides a measure of similarity between the joint pdf of the extracted.(ie.,[5]).

\section{RESULTS}

The figure which is added by noise and the output image which is by passing ICA, PCA, Adaptive PCA and the final graph is as follows:-
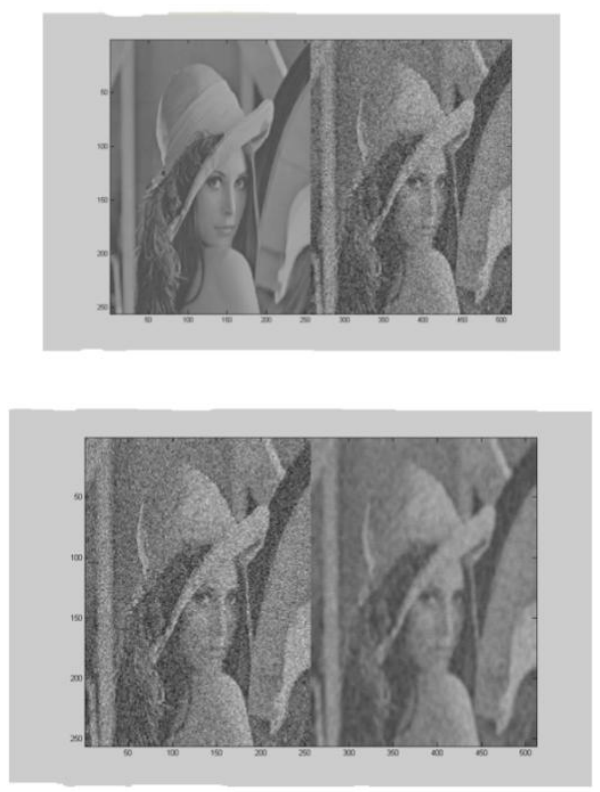

Figure(1) The original + noisy figure Fig(2) Image obtained after adaptive PCA signalsy $=\mathbf{W} \mathbf{x}$ and the joint model pdf of the source signals $\mathbf{s}$. Having such a measure permits us to use standard optimization methods to iteratively update the unmixing matrix in order to maximize this measure of similarity. 

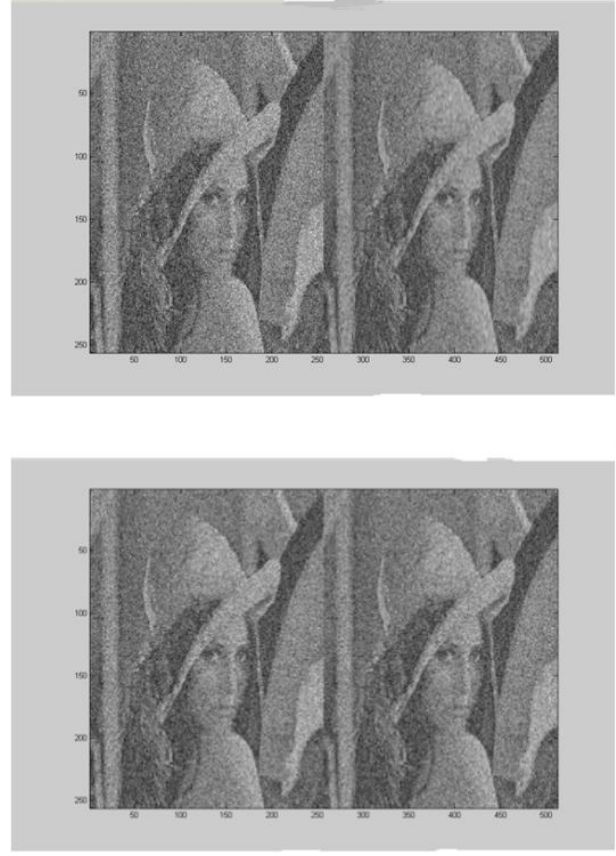

Fig(3) Image obtained after ICA

Fig(4) Image obtained after PCA

\begin{tabular}{|l|l|}
\hline Methods & Signal to noise ratio in $\mathbf{~ d b}$ \\
\hline Original image+noisy image & 8.504725 \\
\hline Local PCA & 30.5415 \\
\hline Fast ICA & 31.9723 \\
\hline Adaptive PCA & 23.9884 \\
\hline
\end{tabular}

\section{ACKNOWLEDGMENT}

Education along with the process of gaining knowledge and stronghold of subject is a continuous and ongoing process. It is an appropriate blend of mindset, learnt skills, experience and knowledge gained from various resources.This project would not have been possible without the support of many people. First and foremost I would like to express my gratitude and indebtedness to my Guide.

\section{REFERENCES}

[1] Potnis Anjali*, Somkuwar Ajay and Sapre S. D. ,A review on natural image denoising using independent component Analysis (ica) technique,Advances in Computational Research, ISSN: 09753273, Volume 2, Issue 1, 2010, pp-06-14

[2] Mukesh c. Motwani, mukesh c gadia, rakhi c. motwani. , Survey of image denoising techniques,

[3] Lecture 3:Principal Component Analysis (PCA).

[4] Xin Li and Michael T. Orchard.,Spatially adaptiveimage denoising under over complete expansion.

[5] JAMES V. STONEIndependent Component Analysis(John Wiley \& Sons, Ltd, Chichester, 2005.)

[6] CeLiu, Student Member, IEEE,RichardSzeliski, Fellow, IEEE, Sing Bing Kang, Senior Member, IEEE, C. Lawrence Zitnick, Member, IEEE, and William T. Freeman, Senior Member, IEEE,Automatic Estimation and Removal of Noise from a Single Image,IEEE TRANSACTIONS ON PATTERN ANALYSIS AND MACHINE INTELLIGENCE, VOL. 30, NO. 2, FEBRUARY 2008.

\section{BIOGRAPHIES}

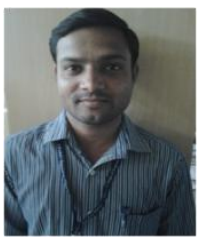

VipulPatil received the B.E. degree in 2010 in Electronics and communication Engineering. Currently he is doing M.E. ( Electronics and communication) from ShriGulabraoDeokar college of Engineering, Jalgaon. His interest in image processing, medical engineering.

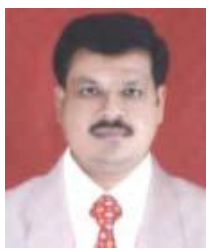

Anil JanardanPatil received his B.E.(Electronics) degree in 1987 from Amravati University, Amravati, India, and M.E.(Industrial Electronics) degree in 1993from Maharaja Sayaji University, Baroda, India. He received his Ph.D. degree in Electronics Engineering in 2002 from AvadheshPratap Sing University, Rewa, M.P., India. Presently, he is working as Principal ogShriGulabraoDeokar College of Engineering, Jalgaon , India. He is member of various professional bodies like IE $\&$ ISTE. His research area includes power electronics and microwave engineering. His work has been published in various international and national journals and conferences including IEEE \& IETE. 